\title{
Automatic Energy-aware Performance Analysis of Mobile Ad-hoc Networks
}

\author{
Lucia Gallina $^{1}$, Tingting Han $^{2}$, Marta Kwiatkowska ${ }^{2}$, Andrea Marin ${ }^{1}$, Sabina Rossi ${ }^{1}$ and Alvise Spanò ${ }^{1}$ \\ ${ }^{1}$ Università Ca’ Foscari Venezia, Italy \\ ${ }^{2}$ University of Oxford, United Kingdom
}

\begin{abstract}
We present a framework to automatically evaluate the performance of Mobile Ad-hoc Networks (MANETs) in terms of different kinds of metrics, such as throughput and energy consumption. We use a probabilistic process calculus to model MANETs; we translate process terms into Markov Decision Processes (MDPs) and use the probabilistic model checker PRISM to automatically evaluate the network performance. We present a case study consisting of a network which uses flooding for communicating, and we analyse how time and energy costs vary when pursuing different power control strategies.

Index Terms-process algebra; MANETS; model checking; network throughput; energy consumption
\end{abstract}

\section{INTRODUCTION}

Mobile ad-hoc networks (MANETs) are systems of mobile devices communicating with each other through wireless links without a pre-established networking infrastructure. The devices may rely on exhaustible power sources, such as batteries, and hence have strict requirements regarding their energy consumption. For these reasons, when dealing with networks of this type, communication protocols have to face the problem of providing full connectivity between network devices while maintaining good performance, both in terms of throughput and of energy conservation (see, e.g., [16], [17]).

We present a framework to automatically evaluate the performance of MANETs in terms of different kinds of metrics. We use the Probabilistic Energy-aware Broadcast Unicast and Multicast (PEBUM) calculus, introduced in [6], to model MANETs. This is a probabilistic process calculus particularly aimed at providing performance analysis in terms of metrics such as energy conservation and throughput.

PRISM [11] is a tool for modelling and analysing systems that exhibit a probabilistic behaviour. It supports, among others, the modelling of Markov Decision Processes (MDPs), where nondeterministic and probabilistic aspects coexist.

In this paper we exploit the PRISM tool to perform automated, quantitative verification and analysis of wireless networks for a range of performance metrics. Specifically, we develop a parser to translate a PEBUM process term, representing a network, into an MDP model expressed in the PRISM language. Moreover, we formulate the metrics for computing the time and energy cost of a network transmission in terms of reward structures.

We demonstrate the effectiveness of our framework on a case study consisting of a MANET which uses flooding to forward messages. We analyse the performance of the communication between two static devices by varying the transmission power of the intermediate, possibly mobile, nodes forwarding the packets within the network. Then we study how the time and energy costs vary when pursuing different power control strategies for the intermediate communications.

Related Work. Probabilistic models are nowadays widely used in the design and verification of complex systems. Bernardo et al. introduced $\mathrm{EMPA}_{g r}$ [2], an extended Markovian process algebra including probabilities, priority and exponentially distributed durations. Palamidessi et al. [8] defined an extension of the applied pi-calculus with nondeterministic and probabilistic choice operators. Among the stochastic process algebras for performance evaluation, one of the most successful is the Performance Evaluation Process Algebra (PEPA), introduced by Hillston in [9], which is used to model systems composed of concurrently active components which cooperate and share work. For both the probabilistic applied pi-calculus and PEPA model checking algorithms built upon the PRISM tool have been developped (see [14], [3]). Although model checking implementations have already been proposed for other process calculi, our work is specifically aimed at studying the performance of mobile ad-hoc and sensor networks. Indeed, we exploit the main features of the PEBUM calculus which allows one to represent variable transmission ranges and probabilistic movements of nodes within the network area.

Regarding energy usage, several papers address the problem of studying the energy consumption of a specific communication protocol for wireless networks. For instance, in [17] the authors define a Markov reward process (see, e.g., [15]) modelling some protocols for pairwise communications. A steady-state quantitative analysis is then obtained, and from this the average performance indices are computed. In [1] Bernardo et al. present a methodology to predict the impact of power management techniques on system functionality and performance. In [16] the authors define a set of metrics to analyse the energy consumption which are then estimated through simulation, and show how some modifications in the protocols can improve their efficiency.

\section{The CAlculus}

We present the PEBUM calculus [6] which models mobile adhoc networks as a collection of nodes, running in parallel and using channels to broadcast messages. It supports multicast communications as well as power control. 
Syntax. We use $c$ for channels, $n$ for nodes, $l$ and $k$ for locations, $r$ for transmission radii; $x$ for variables. Closed values contain nodes, locations, transmission radii and any basic value (booleans, integers, ...). Open values include variables only. We use $u$ and $v$ for closed values, $w$ for open values, and $\tilde{v}$, $\tilde{w}$ for tuples of values. We write Loc for the set of locations.

Networks are defined as the parallel composition of distinct nodes. We write $n[P]_{l}$ for a node $n$, located at the location $l$ and executing the process $P$. We denote by $M_{1} \mid M_{2}$ the parallel composition of two networks and by $\prod_{i \in I} M_{i}$ the parallel composition of the networks $M_{i}$, for $i \in I$. Nodes cannot be created or destroyed. A process $P$ is as follows:

- $\mathbf{0}$ denotes the inactive process;

- $c(\tilde{x}) . P$ can receive a tuple $\tilde{w}$ of closed values via channel $c$ and continue as $P\{\tilde{w} / \tilde{x}\}$, i.e., as $P$ with $\tilde{w}$ substituted for $\tilde{x}$. In the process $c(\tilde{x}) . P$, the variables in $\tilde{x}$ are bound in $P$;

- $\bar{c}_{L, r}\langle\tilde{w}\rangle . P$ can send a tuple of closed values $\tilde{w}$ via channel $c$ and continue as $P$. The tag $L$ is used to maintain the set of observation locations such that $L=L o c$ represents a broadcast transmission, while a finite set of locations $L$ denotes a multicast communication (unicast if $L$ is a singleton). The $\operatorname{tag} r$ represents the power of the transmission. Syntactically, tags $L$ and $r$ may be variables, but they must be instantiated when the output prefix is ready to fire;

- $\left[w_{1}=w_{2}\right] P, Q$ behaves as $P$ if $w_{1}=w_{2}$, as $\mathbf{Q}$ otherwise.

- $A\langle\tilde{w}\rangle$ denotes a process defined via a (possibly recursive) definition $A(\tilde{x}) \stackrel{\text { def }}{=} P$, with $|\tilde{x}|=|\tilde{w}|$ where $\tilde{x}$ contains all channels and variables that appear free in $P$.

A node can be either mobile or static. Each node $n$ is associated with a pair $\left\langle r_{n}, \mathbf{J}^{n}\right\rangle$, where $r_{n}$ is a non negative real number denoting the maximum transmission radius that $n$ can use to transmit, while $\mathbf{J}^{n}$ is the transition probability matrix of a discrete time Markov chain, where $\mathbf{J}_{l k}^{n}$ is the probability that the node $n$ located at $l$, after executing a movement, will be located at $k$. Hence, $\sum_{k \in L o c} \mathbf{J}_{l k}^{n}=1$ for all $l \in L o c$. Notice that, for static nodes, $\mathbf{J}_{l l}^{n}=1$ for $l \in L o c$ and $\mathbf{J}_{l k}^{n}=0$ for $l \neq k$.

Probability distributions. We denote by $\mu_{l}^{n}$ the probability distribution associated with node $n$ located at $l$, that is, a function over $L o c$ such that $\mu_{l}^{n}(k)=\mathbf{J}_{l k}^{n}$, for $k \in L o c$. Moreover, if $M=\prod_{i \in I} n_{i}\left[P_{i}\right]_{l_{i}}$ is a network, then for all $k \in I, \llbracket M \rrbracket_{\mu_{l_{k}}}$ denotes the probability distribution over the set of networks induced by $\mu_{l_{k}}^{n_{k}}$, i.e., for all networks $M^{\prime}$ :

$$
\llbracket M \rrbracket_{\mu_{l_{k}}^{n_{k}}}\left(M^{\prime}\right)= \begin{cases}\mu_{l_{k}}^{n_{k}}\left(l_{k}^{\prime}\right) & \text { if } M^{\prime}=\prod_{i \in I} n_{i}\left[P_{i}\right]_{l_{i}^{\prime}} \\ & \text { for } l_{i}^{\prime}=l_{i} \forall i \neq k \\ 0 & \text { otherwise }\end{cases}
$$

Note that $\llbracket M \rrbracket_{\mu_{l_{k}}^{n_{k}}}\left(M^{\prime}\right)$ is the probability that network $M$ evolves to $M^{\prime}$ due to the movement of the node $n_{k}$ located at $l_{k}$. We say that $M^{\prime}$ is in the support of $\llbracket M \rrbracket_{\mu_{l_{k}}}$ if $\llbracket M \rrbracket_{\mu_{l_{k}}}^{n_{k}}\left(M^{\prime}\right) \neq 0$. We write $\llbracket M \rrbracket_{\Delta}$ for the Dirac distribution on network $M$, namely the probability distribution defined as: $\llbracket M \rrbracket_{\Delta}(M)=1$ and $\llbracket M \rrbracket_{\Delta}\left(M^{\prime}\right)=0$ for all $M^{\prime} \neq M$. We let $\theta$ range over $\left\{\mu_{l}^{n} \mid n\right.$ is a node and $\left.l \in L o c\right\} \cup\{\Delta\}$.
Reduction Semantics. The dynamics of the calculus is specified by the probabilistic reduction relation over networks $(\rightarrow)$, described in Table I. It relies on an auxiliary relation, called structural congruence $(\equiv)$, such that for instance $M \mid N \equiv$ $N|M,(M \mid N)| M^{\prime} \equiv M \mid\left(N \mid M^{\prime}\right)$ and $M \mid \mathbf{0} \equiv M$. The probabilistic reduction relation takes the form $M \rightarrow \llbracket M^{\prime} \rrbracket_{\theta}$, which describes a transition that leaves from a network $M$ and leads to a probability distribution $\llbracket M^{\prime} \rrbracket_{\theta}$.

Rule (R-Bcast) models the transmission of a tuple of messages $\tilde{v}$ to the receivers within the locations in $L$, using channel $c$ and transmission radius $r$. Indeed, nodes communicate using radio frequencies that enable only message broadcasting. However, a node may decide to communicate with a specific set of nodes: depending on $L$, it can be a broadcast, multicast or unicast communication. The tag $r$ indicates the transmission radius required for that communication and may vary due to the energy consumption strategy adopted by the communication protocol. Transmission are non-blocking: they proceed even if there are no nodes listening. The messages transmitted may be received only by those nodes which lie in the transmission area of the sender. Function $d(\cdot, \cdot)$ returns the distance between two locations. Rule (R-Move) states that a node $n$ located at $l$ and executing a moving action will reach a location with the probability described by the distribution $\mu_{l}^{n}$ that depends on the Markov chain $\mathbf{J}^{n}$ associated with $n$. Movements are atomic actions: while moving, a node cannot do anything else. Rules (R-Par) and (R-Struct) are standard.

Observation Semantics. Given a network M, we write $M \rightarrow_{\theta}$ $N$ if $M \rightarrow \llbracket M^{\prime} \rrbracket_{\theta}$ and $N$ is in the support of $\llbracket M^{\prime} \rrbracket_{\theta}$. An execution for $M$ is a (possibly infinite) sequence of steps $M \rightarrow_{\theta_{1}} M_{1} \rightarrow_{\theta_{2}} M_{2} \ldots$. We write last $(e)$ for the final state of a finite execution $e, e^{j}$ for the prefix execution $M \rightarrow_{\theta_{1}}$ $M_{1} \ldots \rightarrow_{\theta_{j}} M_{j}$ of length $j$ of the execution $e=M \rightarrow_{\theta_{1}}$ $M_{1} \ldots \rightarrow_{\theta_{j}} M_{j} \rightarrow_{\theta_{j+1}} M_{j+1} \ldots$. We write $M \rightarrow{ }^{*} M^{\prime}$ if there exists a finite execution $e$ for $M$ such that $\operatorname{last}(e)=M^{\prime}$. We denote by behave $(M)=\left\{\llbracket M^{\prime} \rrbracket_{\theta} \mid M \rightarrow \llbracket M^{\prime} \rrbracket_{\theta}\right\}$ the set of all the distributions $M$ can reach in a single reduction step.

In order to resolve the nondeterminism in a network execution, we use schedulers to choose one distribution from behave $(M)$. Given a finite execution $e$, a scheduler is a total function $F$ assigning a distribution $\llbracket N \rrbracket_{\theta} \in \operatorname{behave}(\operatorname{last}(e))$ to $e$. Given a network $M$ and a scheduler $F$, we define the set of executions starting from $M$ and driven by $F$ as:

$$
\begin{aligned}
\operatorname{Exec}_{M}^{F}= & \left\{e=M \rightarrow \rightarrow_{\theta_{1}} M_{1} \rightarrow{ }_{\theta_{2}} M_{2} \cdots \mid \forall j>0,\right. \\
& M_{j-1} \rightarrow \llbracket M_{j}^{\prime} \rrbracket_{\theta_{j}}, \llbracket M_{j}^{\prime} \rrbracket_{\theta_{j}}=F\left(e^{j-1}\right) \\
& \text { and } \left.\llbracket M_{j}^{\prime} \rrbracket \rrbracket_{\theta_{j}}\left(M_{j}\right)>0\right\} .
\end{aligned}
$$

Formally, given a finite execution $e=M \rightarrow_{\theta_{1}} M_{1} \ldots \rightarrow_{\theta_{k}} M_{k}$ starting from a network $M$ and driven by a scheduler $F$ we define the probability for $M$, following $F$, to perform $e$, as:

$$
P_{M}^{F}(e)=\llbracket M_{1}^{\prime} \rrbracket_{\theta_{1}}\left(M_{1}\right) \cdot \ldots \cdot \llbracket M_{k}^{\prime} \rrbracket_{\theta_{k}}\left(M_{k}\right)
$$

where $\forall 0<j \leq k, \llbracket M_{j}^{\prime} \rrbracket_{\theta_{j}}=F\left(e^{j-1}\right)$.

Given a scheduler $F, \sigma$ Field $M_{M}^{F}$ is the smallest $\sigma$-field on $\operatorname{Exec}_{M}^{F}$ that contains the basic cylinders $e \uparrow$, where $e \in \operatorname{Exec}_{M}^{F}$. The probability measure $\operatorname{Prob}_{M}^{F}$ is the unique 


$$
\begin{aligned}
& \text { (R-Bcast) } \frac{}{n\left[\bar{c}_{L, r}\langle\tilde{v}\rangle \cdot P\right]_{l}\left|\prod_{i \in I} n_{i}\left[c\left(\tilde{x}_{i}\right) \cdot P_{i}\right]_{l_{i}} \rightarrow \llbracket n[P]_{l}\right| \prod_{i \in I} n_{i}\left[P_{i}\left\{\tilde{v}_{i} / \tilde{x}_{i}\right\}\right]_{l_{i}} \rrbracket_{\Delta}} \\
& \text { where } 0<r \leq r_{n}, \forall i \in I . d\left(l, l_{i}\right) \leq r, r_{i}>0 \text { and }\left|\tilde{x}_{i}\right|=\left|\tilde{v}_{i}\right|
\end{aligned}
$$

(R-Move) $\frac{}{n[P]_{l} \rightarrow \llbracket n[P]_{l} \rrbracket_{\mu_{l}^{n}}} \quad$ (R-Par) $\frac{M \rightarrow \llbracket M^{\prime} \rrbracket_{\theta}}{M\left|N \rightarrow \llbracket \llbracket M^{\prime}\right| N \rrbracket_{\theta}} \quad$ (R-Struct) $\frac{M \rightarrow \llbracket M^{\prime} \rrbracket_{\theta} M^{\prime} \equiv N^{\prime}}{M \rightarrow \llbracket N^{\prime} \rrbracket_{\theta}}$

TABLE I: Reduction Semantics

measure on $\sigma$ Field ${ }_{M}^{F}$ such that $\operatorname{Prob}_{M}^{F}(e \uparrow)=P_{M}^{F}(e)$. Given a measurable set of networks $H$, we denote by $\operatorname{Exec}_{M}^{F}(H)$ the set of executions starting from $M$ and reaching a state in $H$. Formally, $\operatorname{Exec}_{M}^{F}(H)=\left\{e \in \operatorname{Exec}_{M}^{F} \mid \operatorname{last}\left(e^{j}\right) \in H\right.$ for some $j$. We denote the probability for a network $M$ to evolve into a network in $H$ according to the policy given by $F$ as $\operatorname{Prob}_{M}^{F}(H)=\operatorname{Prob}_{M}^{F}\left(\operatorname{Exec}_{M}^{F}(H)\right)$.

The notion of barb introduced below denotes an observable transmission with a certain probability according to a fixed scheduler. In our definition, a transmission is observable only if at least one recipient in the set of the observation locations is able to receive the message.

Definition 2.1: [Barb] Let $M \equiv n\left[\bar{c}_{L, r}\langle\tilde{v}\rangle . P\right]_{l} \mid M^{\prime}$. We say that $M$ has a barb on a channel $c$ at locations $K(\neq \emptyset)$, denoted $M \downarrow_{c} @ K$, if $\exists K \subseteq L$ such that $d(l, k) \leqslant r, \forall k \in K$.

Definition 2.2: [Probabilistic Barb] We say that a network $M$ has a probabilistic barb with probability $p$ on a channel $c$ at locations $K$ according to the scheduler $F$, written $M \Downarrow_{p}^{F} c @ K$, if $\operatorname{Prob}_{M}^{F}(H)=p$ where $H=\left\{M^{\prime} \mid M \rightarrow^{*} M^{\prime}, M^{\prime} \downarrow_{c} @ K\right\}$.

Intuitively, for a given network $M$ and scheduler $F$, if $M \Downarrow_{p}^{F} c @ K$ then there is a positive probability that $M$, driven by $F$, performs a transmission on channel $c$ and at least one of the recipients in the observation locations is able to correctly listen to it. Hereafter, we introduce a probabilistic observational congruence, in the style of [8], which is a relation relative to a set of schedulers $\mathcal{F}$ (we call it $\mathcal{F}$-relation) and is defined as the largest $\mathcal{F}$-relation as follows. Let $\mathcal{R}^{\mathcal{F}}$ be a relation over networks and consider the following properties: Barb preservation. $\mathcal{R}^{\mathcal{F}}$ is barb preserving if $M \mathcal{R}^{\mathcal{F}} N$ and $M \Downarrow_{p}^{F} c$ for some $F \in \mathcal{F}$ implies that $\exists F^{\prime} \in \mathcal{F}: N \Downarrow_{p}^{F^{\prime}} c$.

Reduction closure. $\mathcal{R}^{\mathcal{F}}$ is reduction closed if $M \mathcal{R}^{\mathcal{F}} N$ implies: $\forall F \in \mathcal{F}, \exists F^{\prime} \in \mathcal{F}: \forall \mathcal{C} \in \mathcal{N} / \mathcal{R}^{\mathcal{F}}, \operatorname{Prob}_{M}^{F}(\mathcal{C})=\operatorname{Prob}_{N}^{F^{\prime}}(\mathcal{C})$. Contextuality. $\mathcal{R}^{\mathcal{F}}$ is contextual if $M \mathcal{R}^{\mathcal{F}} N$ implies that, $\forall$ context $\mathcal{C}[\cdot], \mathcal{C}[M] \mathcal{R}^{\mathcal{F}} \mathcal{C}[N]$, where $\mathcal{C}[\cdot]::=[\cdot]|[\cdot]| M|M|[\cdot]$.

Definition 2.3: [Probabilistic observational congruence] Probabilistic observational congruence relative to $\mathcal{F}$, written $\cong_{p}^{\mathcal{F}}$, is the largest symmetric $\mathcal{F}$-relation over networks which is reduction closed, barb preserving and contextual.

Two networks are related by $\cong_{p}^{\mathcal{F}}$ if they exhibit the same probabilistic behaviour (communications) relative to the corresponding sets of observable locations and the fixed set of schedulers $\mathcal{F}$. In [7] we have developed a bisimulation-based proof technique for $\cong_{p}^{\mathcal{F}}$.

Cost preorder. In [5] we introduced a preorder, which allows us to compare networks that have the same probabilistic observable behaviours, but different performance in terms of metrics such as energy consumption and time delay. The preorder is based on the definition of a cost function, denoted Cost, which returns for given a network $M$, a set $H$ of goal networks and a scheduler $F$, the cost for $M$ to reach $H$ following $F$. Our cost function can be used to define an efficiency preorder as follows.

Definition 2.4: [Efficiency preorder] Let $\mathcal{H}$ be a countable set of sets of networks and $\mathcal{F}$ be a set of schedulers. We say that $N$ is as least as efficient as $M$ relative to $\mathcal{F}$ and $\mathcal{H}$,

$$
N \sqsubseteq\langle\mathcal{F}, \mathcal{H}\rangle M,
$$

if $N \cong{ }_{p}^{\mathcal{F}} M$ and, for all schedulers $F \in \mathcal{F}$ and for all $H \in \mathcal{H}$, there exists $F^{\prime} \in \mathcal{F}$ such that $\operatorname{Cost}_{N}^{F^{\prime}}(H) \leq \operatorname{Cost}_{M}^{F}(H)$.

\section{A PARSER FOr PEBUM CALCULUS}

In this section we introduce a translator, implemented in F\# [4] with FsYacc and FsLex [12], which compiles PEBUM networks into the PRISM language. Our tool parses an input source file with the description of a network according to the syntax introduced in Section II and translates it into the corresponding MDP for PRISM.

\begin{tabular}{|c|c|}
\hline $\begin{array}{l}\text { Networks } \\
\text { 0 } \\
\mid \begin{array}{l}M_{1} \mid M_{2} \\
n @ l\{P\}\end{array}\end{array}$ & $\begin{array}{l}\text { Processes } \\
\mathbf{0} \\
\mid(\tilde{x}) \leftarrow c ; P \\
\mid \tilde{v} \rightarrow c @ L / r ; P \\
\text { if } w_{1}=w_{2} \text { then } P \text { else } Q \\
\operatorname{rec} P\end{array}$ \\
\hline
\end{tabular}

Input of the parser. The input of the parser contains:

- the PEBUM network written in the following grammar:

- a transition probability matrix $\mathbf{J}^{n}$ associated with each node $n$ as illustrated below, where $\mathrm{L}_{1}, \ldots, \mathrm{L}_{\mathrm{k}}$ are the locations in the network and $p_{i, j}$ is the probability for the node $n$ to move from the location $\mathrm{L}_{i}$ to $\mathrm{L}_{j}$.

$$
\begin{aligned}
& \mathbf{J}^{n}=\begin{array}{lllll}
\mathrm{L}_{1} & \mathrm{~L}_{2} & \ldots & \mathrm{L}_{\mathrm{k}}
\end{array} \\
& \begin{array}{llll}
\mathrm{p}_{1,1} & \mathrm{p}_{1,2} & \ldots & \mathrm{p}_{1, \mathrm{k}}
\end{array} \\
& \begin{array}{llll}
\mathrm{p}_{\mathrm{k}, 1} & \mathrm{p}_{\mathrm{k}, 2} & \ldots & \mathrm{p}_{\mathrm{k}, \mathrm{k}}
\end{array}
\end{aligned}
$$

- a triangular matrix Dist, describing the distances between each pair of network locations. The matrix is triangular because we assume the distance to be symmetric. 


$$
\text { Dist }=\begin{array}{cccc}
\mathrm{L}_{1} & \mathrm{~L}_{2} & \ldots & \mathrm{L}_{\mathrm{k}} \\
\mathrm{d}_{1,1} & \mathrm{~d}_{1,2} & \ldots & \mathrm{d}_{1, \mathrm{k}} \\
& & \ldots & \\
& & & \mathrm{d}_{\mathrm{k}, \mathrm{k}}
\end{array}
$$

Output of the parser. The output of the parser is an MDP written in the PRISM language [11]. As an example, Fig. 1 shows the translation of the simple PEBUM network

$$
n_{1}\left[\bar{c}_{l_{1}, r}\langle m s g 1\rangle . c(x)\right]_{l_{1}} \mid n_{2}\left[c(y) \cdot \bar{c}_{l_{1}, r}\langle m s g 2\rangle\right]_{l_{2}} .
$$

Constants are used to represent the set $L o c$ of locations as well as the set of messages sent by the network nodes during the execution. Given a network $M \equiv \prod_{i \in I} n_{i}\left[P_{i}\right]_{l_{i}}$, each network node $n_{i}\left[P_{i}\right]_{l_{i}}$ corresponds to an MDP module, with name $n_{i}$. The variable $l o c_{n_{i}}$ refers to the current location of $n_{i}$; the variable $\operatorname{proc}_{n_{i}}$ represents the current process status, which is used to control the order of process execution (since processes are deterministic and sequential). If the process executed by the node contains bound variables, they will be defined in the MDP too (see variable $x$ in the example in Fig. 1).

Modules may execute commands (or actions) corresponding to the reduction rules (R-Bcast) and (R-Move) in Table I:

- (R-Bcast) $\quad n\left[\bar{c}_{L, r}\langle\tilde{v}\rangle \cdot P\right]_{l} \mid \prod_{i \in I} n_{i}\left[c\left(\tilde{x}_{i}\right) \cdot P_{i}\right]_{l_{i}}$

$$
\rightarrow \llbracket n[P]_{l} \mid \prod_{i \in I} n_{i}\left[P_{i}\left\{\tilde{v}_{i} / \tilde{x}_{i}\right\}\right]_{l_{i}} \rrbracket_{\Delta}
$$

Since in PRISM synchronisations are modelled by labelling commands with actions, we associate the following action label with each message transmission:

$$
\left[\mathrm{c} \_\mathrm{n}_{1} \ldots \mathrm{n}_{\mathrm{h} 1} \_\mathrm{v}_{1} \ldots \mathrm{v}_{\mathrm{h} 2} \_\mathrm{K}_{1} \_\ldots \_\mathrm{K}_{\mathrm{h} 3} \_\mathrm{R}_{1} \ldots \_\mathrm{R}_{\mathrm{h} 4}\right]
$$

where $\left\{\mathrm{n}_{1}, \ldots, \mathrm{n}_{\mathrm{h} 1}\right\}$ is the set of the nodes $n_{i}(i \in I)$ receiving the message; $\left(\mathrm{v}_{1}, \ldots, \mathrm{v}_{\mathrm{h} 2}\right)$ is the tuple $\tilde{v}$ of messages; $\left\{R_{1}, \ldots, R_{\mathrm{h}}\right\}$ is the set of locations whose distance from $l$ is less than the radius; $\left\{\mathrm{K}_{1}, \ldots, \mathrm{K}_{\mathrm{h} 3}\right\}$ is the intersection between the set of the observation locations and the set $\left\{R_{1}, \ldots, R_{h 4}\right\}$.

The module of sender $n$ contains the following command in PRISM (for $L_{k}=l$ ):

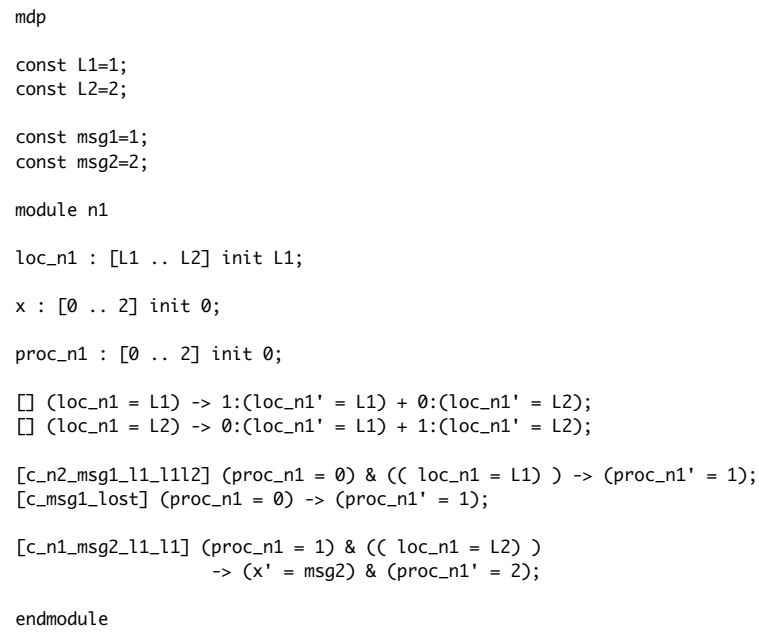

Fig. 1: PRISM module for $n_{1}$

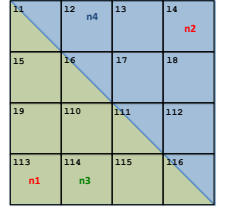

(a) Scheme 1

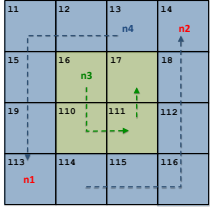

(b) Scheme 2
Fig. 2: The different network schemes

$\left[\mathrm{c} \_\mathrm{n}_{1} \ldots \mathrm{n}_{\mathrm{h} 1} \_\mathrm{v}_{1} \ldots \mathrm{v}_{\mathrm{h} 2 \_} \mathrm{K}_{1} \ldots \ldots \_\mathrm{K}_{\mathrm{h} 3} \mathrm{R}_{1} \ldots \mathrm{R}_{\mathrm{h} 4}\right]$ (proc_n=counter) $\&\left(\right.$ loc_n $\left.=\mathrm{L}_{\mathrm{k}}\right) \rightarrow\left(\right.$ proc_n' $\mathrm{n}^{\prime}$ counter +1$)$;

The module of a receiver node $n_{i}(i \in I)$ contains the following command in PRISM:

$$
\begin{aligned}
& {\left[\mathrm{c} \_\mathrm{n}_{1} \ldots \mathrm{n}_{\mathrm{h} 1} \_\mathrm{v}_{1} \ldots \mathrm{v}_{\mathrm{h} 2} \_\mathrm{K}_{1} \_\ldots \_\mathrm{K}_{\mathrm{h} 3} \mathrm{R}_{1} \ldots \mathrm{R}_{\mathrm{h} 4}\right]} \\
& (\text { proc_n } n=\text { counter }) \&\left(\left(\text { loc_n } n_{i}=R_{1}\right)|\cdots|\left(\text { loc_n } n_{i}=R_{k}\right)\right) \\
& \rightarrow\left(\text { proc_n } \mathrm{n}_{\mathrm{i}}^{\prime}=\text { counter }+1\right) \&\left(\mathrm{x}_{\mathrm{i}}=\mathrm{msg}\right) \text {; }
\end{aligned}
$$

If $I$ is the empty set, then it means that no nodes receive the transmission, and the message is lost. We add a command with a loss-message action to the sender's module:

$$
\begin{aligned}
& \text { [c_v } \left.1 \ldots \mathrm{v}_{\mathrm{h} 2 \_ \text {lost }}\right](\text { proc_n }=\text { counter }) \&\left(\text { loc_n }=\mathrm{L}_{\mathrm{k}}\right) \\
& \left.\rightarrow \text { (proc_n' }{ }^{\prime}=\text { counter }+1\right) \text {; } \\
& \text { - (R-Move) } \quad n[P]_{l} \rightarrow \llbracket n[P]_{l} \rrbracket_{\mu_{l}^{n}}
\end{aligned}
$$

In PRISM the mobility of nodes is expressed using a different command for each row of matrix $\mathbf{J}^{n}$ associated with each node. In particular, each $i$-th row of matrix $\mathbf{J}^{n}$ corresponds to the command (for $\mathrm{L}_{1}, \ldots, \mathrm{L}_{j} \in L o c$ ):

$$
\text { [] }\left(\text { loc_n }=\mathrm{L}_{\mathrm{i}}\right) \rightarrow \mathrm{p}_{\mathrm{i}, 1}:\left(\text { loc_n' } \mathrm{n}^{\prime}=\mathrm{L}_{1}\right)+\cdots+\mathrm{p}_{\mathrm{i}, \mathrm{j}}\left(\text { loc_n } \mathrm{n}^{\prime}=\mathrm{L}_{j}\right) \text {; }
$$

The parallel composition ( $\mathrm{R}$ - Par) is modelled by the nondeterministic choices of the modules to execute synchronising or non-synchronising (message losses) commands.

\section{A CASE STUdY}

We present a case study to show the effectiveness of translating our process algebra into an MDP in order to automatise the performance analysis for a range of metrics using PRISM.

The network. We consider two static nodes ( $n_{1}$ and $\left.n_{2}\right)$ communicating in a $60 \times 60$ metres network area (see Fig. 2). The distances between cells are determined by considering the centre of each cell and calculating the euclidean distance between each pair of centres (each cell is $15 \times 15$ metres). Since the two static nodes are too distant to reach each other (compared to their radius 20), the communication is possible only if there are other intermediate nodes inside the network forwarding the messages between $n_{1}$ and $n_{2}$. We consider a simple flooding algorithm in which mobile nodes forward the messages in lazy mode, i.e., they store the data to be sent until there is another node ready to receive it.

Scheme 1: The first network we consider has two mobile nodes able to forward messages. As shown in Fig. 2.(a), one of the two intermediate nodes can move in the triangular area around the position of $n_{1}$, while the other one covers the triangular area around $n_{2}$. 
Scheme 2: In the second scheme, Fig. 2.(b), one node can only move inside the central area of the network (4 cells), while the second one can only move in the 12 border cells.

We study the performance of these networks by varying the transmission power of the intermediate nodes, in order to find the best power strategy which optimises the performance in terms of energy consumption and throughput. The networks only differ in the transition probability matrices associated with each node, while their behaviour is expressed in the PEBUM calculus as follows where $R A D \in\{20,30,40,50\}$ :

$$
\begin{aligned}
& M_{R A D} \equiv \prod_{i=1}^{4} n_{i}\left[P_{i}\right]_{l_{i}} \text { where: } \\
& \text { - } P_{1} \equiv \bar{c}_{l 4,20}\langle\text { msg_for_n2 }\rangle \cdot P_{1}^{\prime} \\
& P_{1}^{\prime} \equiv c\left(x_{1}\right) \cdot\left[x_{1}=\text { ack_to_n1 }\right] \bar{d}_{l 13,20}\langle\text { ok }\rangle, P_{1}^{\prime} ; \\
& \text { - } P_{2} \equiv c\left(x_{2}\right) \cdot\left[x_{2}=\text { msg_to_n2 }\right] \bar{c}_{l 13,20}\langle\text { ack_to_n1 }\rangle, P_{2} \\
& \text { - } P_{i} \equiv c\left(x_{i}\right) \cdot \bar{c}_{\emptyset, R A D}\left\langle x_{i}\right\rangle . P_{i}, \quad i \in\{3,4\}
\end{aligned}
$$

First we prove that, by varying the value of $R A D$, the probabilistic observation behaviour of $M_{R A D}$ does not change.

Theorem 4.1: For $R 1, R 2 \in\{20,30,40,50\}$,

$$
M_{R 1} \cong \mathcal{F}_{p}^{\mathcal{M}} M_{R 2} \text {. }
$$

For each scheme we build a PRISM MDP, assigning to the variable $R A D$ the values in the set $\{20,30,40,50\}$, in order to investigate how different transmission powers influence the performance of the network.

Costs. We study the energy spent in the transmissions assuming that the cost for the control packets is negligible. We consider the restricted set of schedulers $\mathcal{F} \in$ Sched such that nodes always perform synchronisations where possible and they transmit a message only if at least one node is listening to the channel (i.e., messages are not lost).

We assume quasi-linear cost functions of the form

$$
\sum_{k} w_{k} \cdot \Phi_{k}(-)
$$

that are sums of arbitrary functions $\Phi_{k}\left({ }_{-}\right)$, each of which maps one metric (e.g., energy cost and throughput) to some common measure. In general, we give a different weight $w_{k} \geq 0$ to each $\Phi_{k}\left({ }_{-}\right)$to reflect its importance. We assume that $\sum_{k} w_{k}=1$.

In the following, we first introduce the energy cost $\Phi_{\mathbf{E}}$, then the time cost $\Phi_{\mathbf{T}}$ and finally the global cost function.

Energy cost is defiend as follows:

$$
\mathbf{E}(M, N)=\left\{\begin{array}{c}
\left(\mathrm{En}_{\text {elec }} \times \text { packet_len }+\right. \\
\left.\mathrm{En}_{\text {ampl }} \times \text { packet_len } \times r^{2}\right) \\
\quad \text { if } M \rightarrow_{\theta} N, M \equiv n\left[\bar{c}_{L, r}\langle\tilde{v}\rangle . P\right]_{l} \mid M^{\prime} \\
\quad N \equiv n[P]_{l} \mid N^{\prime} \text { for some } c, L, \tilde{v}, l \\
0 \quad \text { otherwise. }
\end{array}\right.
$$

where $\operatorname{En}_{\text {elec }}(n J / b)$ is the energy dissipated to run the transmitter circuit, $\mathrm{En}_{a m p l}\left(p J / b / m^{2}\right)$ is the Radio amplifier energy. They are constants given a priori (see [13]).
Time cost is defined as follows:

$$
\mathbf{T}(M, N)=\left\{\begin{array}{c}
\text { (packet_len/bandwith) } \\
\text { if } M \rightarrow_{\Delta} N, M \equiv n\left[\bar{c}_{L, r}\langle\tilde{v}\rangle . P\right]_{l} \mid M^{\prime} \\
\text { and } N \equiv n[P]_{l} \mid N^{\prime} \text { for some } c, L, v, l \\
(\text { cell_size/velocity) } \\
\text { if } M \rightarrow \mu_{l}^{n} N, M \equiv n[P]_{l} \mid M^{\prime} \\
\text { and } N \equiv n[P]_{k} \mid M^{\prime}
\end{array}\right.
$$

where cell_size $(\mathrm{m})$ is the size of a cell, velocity $(\mathrm{m} / \mathrm{s})$ is the velocity of the devices and bandwith $(M B)$ is the channel bandwidth of the network. Again they are constants.

Let $\mathcal{X} \in\{\mathbf{E}, \mathbf{T}\}$ be one of the two metrics above, and $e=$ $M_{0} \rightarrow_{\theta_{1}} M_{1} \rightarrow_{\theta_{2}} M_{2} \cdots \rightarrow_{\theta_{k}} M_{k}$ be an execution. We define

$$
\mathcal{X}(e)=\sum_{i=1}^{k} \mathcal{X}\left(M_{i-1}, M_{i}\right) .
$$

Let $H$ be a set of networks. We denote by $\operatorname{Paths}_{M}^{F}(H)$ the set of all executions from $M$ ending in $H$ and driven by scheduler $F$ which are not prefix of any other execution ending in $H$. More formally, Paths ${ }_{M}^{F}(H)=\left\{e \in \operatorname{Exec}_{M}^{F}(H)\right.$ last $(e) \in H$ and $\forall e^{\prime}$ with $\left.e<_{\text {prefix }} e^{\prime}, e^{\prime} \notin \operatorname{Paths}_{M}^{F}(H)\right\}$.

Definition 4.2: Let $H$ be a set of networks and $\mathcal{X} \in$ $\{\mathbf{E}, \mathbf{T}\}$. The average energy and time cost of reaching $H$ from $M$ according to the scheduler $F$ is

$$
\mathcal{X}_{M}^{F}(H)=\frac{\sum_{e \in \operatorname{Paths}_{M}^{F}(H)} \mathcal{X}(e) \times \operatorname{Prob}_{M}^{F}(e)}{\sum_{e \in \operatorname{Paths}_{M}^{F}(H)} \operatorname{Prob}_{M}^{F}(e)} .
$$

Now consider Eq. (1). Let $\Phi_{E}=\mathbf{E}$ and $\Phi_{T}=\mathbf{T}$, and $w$ be the weight of the energy function. We define the cost of reaching a set $H$ from the network $M$ according to $F$ as:

$$
\operatorname{Cost}_{M}^{F}(H)=\alpha \mathbf{E}_{M}^{F}(H)+\beta \mathbf{T}_{M}^{F}(H),
$$

where $\alpha$ and $\beta$ are weighting coefficients expressed in $s^{-1}$ and $J^{-1}$ respectively, and henceforth we simply consider $\alpha=w$ and $\beta=1-w$, with $w \in[0,1]$. In our PRISM MDP the constants are given as follows:

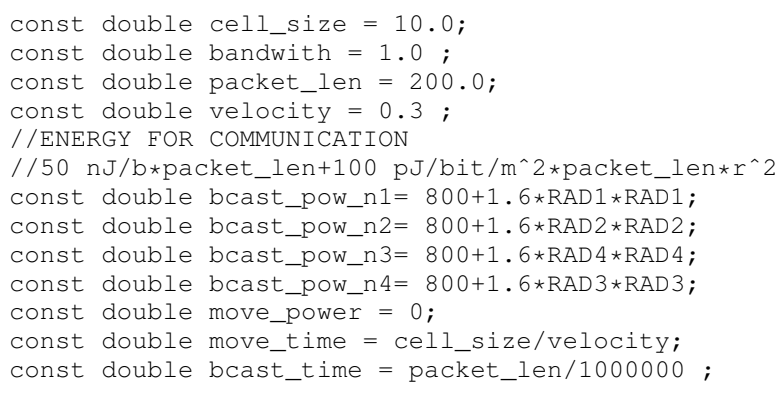

where bcast_pow_ni and bcast_time are the energy and time spent for a single transmission performed by $n_{i}$, while move_power and move_time are the energy and time spent for each single movement step. The cost can be calculated by:

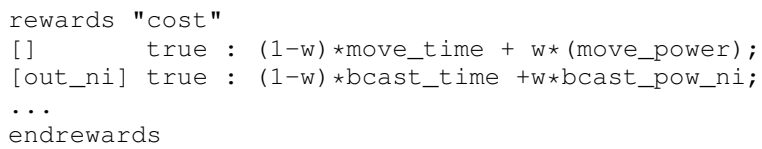




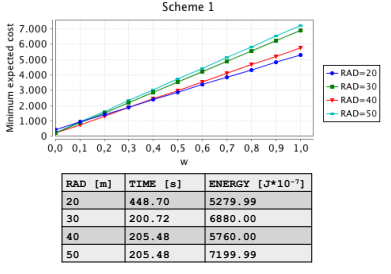

(a) Scheme 1

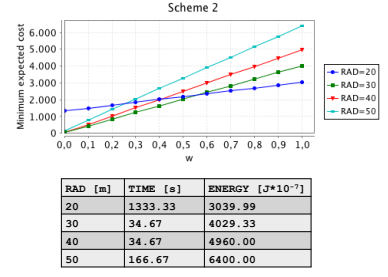

(b) Scheme 2
Fig. 3: Cost results for each scheme

We use the PRISM tool to automatically verify the second point of Def. 2.4, by calculating, for each radius in the set $\{20,30,40,50\}$, the minimum cost among all schedulers under which the goal state is reached with probability 1:

$R\{$ "cost" $\}$ min=? [F goal_state $]$

Fig. 3 specifies, for each scheme, both the time and energy costs and the general cost function (time cost is expressed in $s$ while energy is expressed in $J \times 10^{-7}$ ). First we notice that in both scheme, the smallest radius has the minimum energy cost, and the highest time cost. For $0<w<1$ in general, we investigate for each scheme how the total cost is affected by choosing different power strategies (i.e., transmission radii).

Scheme 1: In Scheme 1 (Fig. 3.(a)), the smallest (largest) radius still seems to give the best (worst) cost. However, using radius 40 is much better than 30 due to the mobility behaviours associated with the intermediate nodes: with radius $30, n_{3}$ is only able to reach $n_{1}$, while $n_{4}$ can only forward $n_{2}$ communications: this situation enlarges the minimum number of transmissions allowing $n_{1}$ and $n_{2}$ to complete their communications. Conversely, choosing a larger radius (40) enables $n_{3}$ (respectively $n_{4}$ ) to eventually reach $n_{2}$ (respectively $n_{1}$ ), drastically reducing the number of transmissions. Since the energy cost using radius 40 is not much higher than the cost spent using radius 20 , while the time costs are halved, we can conclude that for the first scheme the best power management strategy for time-aware applications is to use radius 40 .

Scheme 2: Let us now consider the second scheme (Fig. 3.(b)). With $w \leq 0.57$ the best solution is radius 30, while with $w>0.57$ the best strategy is radius 20. Moreover, if we are interested only in reducing the time spent to communicate, radius 40 is the best choice, since the costs are consistently reduced with respect to the other radii, while radius 20 critically increases time cost. Generally speaking, the best solution is to use radius 30 . We notice that 30 is the smallest distance that the inner area (4 cells) and the outer area (12 cells) can always directly reach each other, while radius 20 never allows $n_{3}$ to reach $n_{1}$ and $n_{2}$. For these reasons time cost consistently increases for radius 20 , while with radius 30 is better (about $\frac{34.67}{1333.33}=2.5 \%$ of the time cost for radius $20)$. With respect to radii 40 and 50 , radius 30 allows one to reduce the energy cost, without deteriorating the time delays.

\section{CONCLUSiOn AND Future WORK}

This paper presented an automatic tool, based on the PEBUM calculus [5], [6], for comparing different costs of networks having the same probabilistic observable behaviour. The automatic quantitative verification of a network has been implemented by model checking. In particular we provided a translation of the PEBUM models into PRISM MDP models [10], which allows one to automatically verify the performance of networks in terms of various metrics.

\section{REFERENCES}

[1] A. Acquaviva, A. Aldini, M. Bernardo, A. Bogliolo, E. Bontà, and E. Lattanzi. A methodology based on formal methods for predicting the impact of dynamic power management. In Formal Methods for Mobile Computing, volume 3465 of lncs, pages 51-58. Springer Berlin / Heidelberg, 2005.

[2] M. Bernardo and M. Bravetti. Performance measure sensitive congruences for Markovian process algebras. Theoretical Computer Science, 290(1):117-160, 2003.

[3] F. Ciocchetta, S. Gilmore, M. L. Guerriero, and J. Hillston. Integrated simulation and model-checking for the analysis of biochemical systems. In Proc. 3rd International Workshop on Practical Applications of Stochastic Modelling (PASM'08), volume 232 of ENTCS, pages 17-38, 2008.

[4] F. DeRemer and T. Pennello. Efficient computation of lalr(1) look-ahead sets. ACM Trans. Program. Lang. Syst., 4(4):615-649, October 1982.

[5] L. Gallina, S. Hamadou, A. Marin, and S. Rossi. A framework for throughput and energy efficiency in mobile ad hoc networks. In Proc. of IFIP Wireless Days 2011. IEEE Press, 2011.

[6] L. Gallina, S. Hamadou, A. Marin, and S. Rossi. A probabilistic energy-aware model for mobile ad-hoc networks. In Proc. of the 18th International Conference on Analytical and Stochastic Modelling Techniques and Applications (ASMTA'11), volume 6751 of LNCS, pages 316-330. Springer-Verlag, 2011.

[7] L. Gallina, S. Hamadou, A. Marin, and S. Rossi. Connectivity and energy aware preorders for mobile ad hoc networks. Technical Report DAIS-2012-1, University Ca' Foscari Venice, 2012.

[8] J. Goubault-Larrecq, C. Palamidessi, and A. Troina. A probabilistic applied pi-calculus. In Proc. of the 5th Asian Symposium on Programming Languages and Systems (APLAS'07), volume 4807/2009 of LNCS, pages 175-190. Springer-Verlag, 2007.

[9] J. Hillston. A Compositional Approach to Performance Modelling. Cambridge University Press, 1996.

[10] A. Hinton, M. Kwiatkowska, G. Norman, and D. Parker. Prism: A tool for automatic verification of probabilistic systems. In Proc. of the 12th International Conference on Tools and Algorithms for the Construction and Analysis of Systems (TACAS'06), volume 3920 of LNCS, pages 441444. Springer-Verlag, 2006.

[11] M. Z. Kwiatkowska, G. Norman, and D. Parker. Prism 4.0: Verification of probabilistic real-time systems. In Ganesh Gopalakrishnan and Shaz Qadeer, editors, CAV, volume 6806 of Lecture Notes in Computer Science, pages 585-591. Springer, 2011.

[12] M.E. Lesk. Lex - a lexical analyzer generator. Computer Science Technical Report 39, Murray Hill, New Jersey: Bell Laboratories, 1975.

[13] T. Venu Madhav and N.V.S.N. Sarma. Maximizing network lifetime through varying transmission radii with energy efficient cluster routing algorithm in wireless sensor networks. International Journal of Information and Electronics Engineering, 2(2):205-209, 2012.

[14] G. Norman, C. Palamidessi, D. Parker, and P. Wu. Model checking probabilistic and stochastic extensions of the $\pi$-calculus. IEEE Transactions on Software Engineering, 35(2):209-223, 2009.

[15] S. M. Ross. Stochastic Processes. John Wiley \& Sons, 2nd edition, 1996.

[16] S. Singh, M. Woo, and C.S. Raghavendra. Power-aware routing in mobile ad hoc networks. In Proc. of the 4th annual ACM/IEEE International Conference on Mobile Computing and Networking (MobiCom'98), pages 181-190. ACM, 1998.

[17] M. Zorzi and R.R. Rao. Error control and energy consumption in communications for nomadic computing. IEEE Transactions on Computers, 46(3):279-289, 1997. 